\title{
ON THE GENERALIZED BI-PERIODIC FIBONACCI AND LUCAS QUATERNIONS
}

\section{YOUNSEOK CHOO}

Received 11 April, 2019

\begin{abstract}
In this paper we introduce the generalized bi-periodic Fibonacci and Lucas quaternions which are the further generalizations of the bi-periodic Fibonacci and Lucas quaternions considered in the literature. For those quaternions, we derive the generating functions, Binet's formulas and Catalan's identities.
\end{abstract}

2010 Mathematics Subject Classification: 11B39; 11B37; 11B52

Keywords: generalized bi-periodic Fibonacci sequence, generalized bi-periodic Lucas sequence, quaternion, Binet's formula, generating function, Catalan's identity

\section{INTRODUCTION}

As is well known, the Fibonacci sequence $\left\{F_{n}\right\}$ is generated from the recurrence relation $F_{n}=F_{n-1}+F_{n-2}(n \geq 2)$ with $F_{0}=0, F_{1}=1$, and the Lucas sequence $\left\{L_{n}\right\}$ is generated from the recurrence relation $L_{n}=L_{n-1}+L_{n-2}(n \geq 2)$ with $L_{0}=2, L_{1}=1$. The Binet's formulas for $\left\{F_{n}\right\}$ and $\left\{L_{n}\right\}$ are respectively given by

$$
\begin{aligned}
& F_{n}=\frac{\alpha^{n}-\beta^{n}}{\alpha-\beta}, \\
& L_{n}=\alpha^{n}+\beta^{n},
\end{aligned}
$$

where $\alpha(>0)$ and $\beta(<0)$ are roots of the equation $x^{2}-x-1=0$.

Many authors generalized the Fibonacci and Lucas sequences by changing initial conditions and/or recurrence relations. In particular, Edson and Yayenie [5] introduced the bi-periodic Fibonacci sequence $\left\{p_{n}\right\}$ defined by

$$
p_{0}=0, p_{1}=1, \quad p_{n}=\left\{\begin{array}{ll}
a p_{n-1}+p_{n-2}, & \text { if } n \text { is even } \\
b p_{n-1}+p_{n-2}, & \text { if } n \text { is odd }
\end{array} \quad(n \geq 2) .\right.
$$

The Binet's formula for $\left\{p_{n}\right\}$ is given by [5]

$$
p_{n}=\frac{a^{\zeta(n+1)}}{(a b)^{\left\lfloor\frac{n}{2}\right\rfloor}}\left(\frac{\alpha^{n}-\beta^{n}}{\alpha-\beta}\right),
$$


where $\alpha(>0)$ and $\beta(<0)$ are roots of the equation $x^{2}-a b x-a b=0$, and $\zeta(\cdot)$ is the parity function such that $\zeta(n)=0$ if $n$ is even and $\zeta(n)=1$ if $n$ is odd.

The bi-periodic Fibonacci sequence $\left\{p_{n}\right\}$ given in (1.1) includes many sequences as special cases. For $a=b=1,\left\{p_{n}\right\}$ becomes the Fibonacci sequence. For $a=b=$ $2,\left\{p_{n}\right\}$ becomes the Pell sequence. If $a=b=k$, then $\left\{p_{n}\right\}$ denotes the $k$-Fibonacci sequence defined in [8], etc.

On the other hand, Bilgici [2] generalized the Lucas sequence by introducing the bi-periodic Lucas sequence $\left\{u_{n}\right\}$ defined by

$$
u_{0}=2, u_{1}=a, u_{n}=\left\{\begin{array}{ll}
b u_{n-1}+u_{n-2}, & \text { if } n \text { is even } \\
a u_{n-1}+u_{n-2}, & \text { if } n \text { is odd }
\end{array} \quad(n \geq 2) .\right.
$$

If $a=b=1$, then $\left\{u_{n}\right\}$ becomes the Lucas sequence $\left\{L_{n}\right\}$. If $a=b=k$, then $\left\{u_{n}\right\}$ becomes the $k$-Lucas sequence in [7].

The Binet's formula for $\left\{u_{n}\right\}$ is given by [2]

$$
u_{n}=\frac{a^{\zeta(n)}}{(a b)^{\left\lfloor\frac{n+1}{2}\right\rfloor}}\left(\alpha^{n}+\beta^{n}\right),
$$

where $\alpha$ and $\beta$ are as defined in (1.2).

A quaternion $q$ is defined by

$$
q=q_{0} e_{0}+q_{1} e_{1}+q_{2} e_{2}+q_{3} e_{3},
$$

where $q_{0}, q_{1}, q_{2}, q_{3} \in \mathbb{R}, e_{0}=1$, and $e_{1}, e_{2}$ and $e_{3}$ are the standard basis in $\mathbb{R}^{3}$ such that $e_{i}^{2}=-1, i=1,2,3$, and

$$
e_{1} e_{2}=-e_{2} e_{1}=e_{3}, e_{2} e_{3}=-e_{3} e_{2}=e_{1}, e_{3} e_{1}=-e_{1} e_{3}=e_{2} .
$$

As noted in the literature $[1,6,9,20]$, quaternions are widely used in the fields of engineering and physics as well as mathematics, and attracted sustained attention from many researchers. In particular, a variety of results are available in the literature on the properties of quaternions related to the sequences described earlier. Horadam [13] defined the Fibonacci quaternion sequence $\left\{G_{n}\right\}$ and Lucas quaternion sequence $\left\{H_{n}\right\}$ as

$$
\begin{aligned}
& G_{n}=F_{n} e_{0}+F_{n+1} e_{1}+F_{n+2} e_{2}+F_{n+3} e_{3}, \\
& H_{n}=L_{n} e_{0}+L_{n+1} e_{1}+L_{n+2} e_{2}+L_{n+3} e_{3},
\end{aligned}
$$

where $F_{n}$ and $L_{n}$ are respectively the $n$th Fibonacci and Lucas numbers.

Following the work of Horadam [13], diverse results have appeared in the literature. Halici [10] obtained the generating functions, Binet's formulas and some combinatorial properties of the Fibonacci and Lucas quaternions. Halici [11] also introduced the complex Fibonacci quaternions. Ramirez [15] studied the properties of the $k$-Fibonacci and $k$-Lucas quaternions. Çimen and İpek [4], Szynal-Liana and Włoch [17] investigated the Pell and Pell-Lucas quaternions. Szynal-Liana and Włoch [17] 
introduced the Jacobsthal and Jacobsthal-Lucas quaternions also. Catarino [3] considered the modified Pell and modified $k$-Pell quaternions. Halici and Karatas [12] defined a general quaternion which includes several quaternions mentioned above as special cases.

Recently Tan et al. [19] introduced the bi-periodic Fibonacci quaternion sequence $\left\{P_{n}\right\}$ defined by

$$
P_{n}=p_{n} e_{0}+p_{n+1} e_{1}+p_{n+2} e_{2}+p_{n+3} e_{3},
$$

where $p_{n}$ is the $n$th bi-periodic Fibonacci number.

The Binet's formula for $\left\{P_{n}\right\}$ is given by [19]

$$
P_{n}= \begin{cases}\frac{1}{(a b)^{\left\lfloor\frac{n}{2}\right\rfloor}}\left(\frac{\alpha^{*} \alpha^{n}-\beta^{*} \beta^{n}}{\alpha-\beta}\right), & \text { if } n \text { is even } \\ \frac{1}{(a b)^{\left\lfloor\frac{n}{2}\right\rfloor}}\left(\frac{\alpha^{* *} \alpha^{n}-\beta^{* *} \beta^{n}}{\alpha-\beta}\right), & \text { if } n \text { is odd }\end{cases}
$$

where $\alpha$ and $\beta$ are as defined in (1.2), and

$$
\begin{aligned}
\alpha^{*} & =\sum_{l=0}^{3} \frac{a^{\zeta(l+1)}}{(a b)^{\left\lfloor\frac{l}{2}\right\rfloor}} \alpha^{l} e_{l}, \\
\beta^{*} & =\sum_{l=0}^{3} \frac{a^{\zeta(l+1)}}{(a b)^{\left\lfloor\frac{l}{2}\right\rfloor}} \beta^{l} e_{l}, \\
\alpha^{* *} & =\sum_{l=0}^{3} \frac{a^{\zeta(l)}}{(a b)^{\left\lfloor\frac{l+1}{2}\right\rfloor}} \alpha^{l} e_{l}, \\
\beta^{* *} & =\sum_{l=0}^{3} \frac{a^{\zeta(l)}}{(a b)^{\left\lfloor\frac{l+1}{2}\right\rfloor}} \beta^{l} e_{l} .
\end{aligned}
$$

Tan et al. [18] also introduced the bi-periodic Lucas quaternion sequence $\left\{U_{n}\right\}$ as follows:

$$
U_{n}=u_{n} e_{0}+u_{n+1} e_{1}+u_{n+2} e_{2}+u_{n+3} e_{3},
$$

where $u_{n}$ is the $n$th bi-periodic Lucas number.

The Binet's formula for $\left\{U_{n}\right\}$ is given by [18]

$$
U_{n}= \begin{cases}\frac{1}{(a b)^{\left\lfloor\frac{n+1}{2}\right\rfloor}}\left(\alpha^{* *} \alpha^{n}+\beta^{* *} \beta^{n}\right), & \text { if } n \text { is even } \\ \frac{1}{(a b)^{\left\lfloor\frac{n+1}{2}\right\rfloor}}\left(\alpha^{*} \alpha^{n}+\beta^{*} \beta^{n}\right), & \text { if } n \text { is odd }\end{cases}
$$

where $\alpha, \beta$ are as defined in (1.2), and $\alpha^{*}, \beta^{*}, \alpha^{* *}$ and $\beta^{* *}$ are as defined in (1.6).

If we use the initial condition $P_{0}=e_{1}+e_{2}+2 e_{3}$ and $P_{1}=e_{0}+e_{1}+2 e_{2}+3 e_{3}$ in (1.5), then $\left\{P_{n}\right\}$ is the same as the generalized Fibonacci quaternion sequence considered in [14]. Also if we set $P_{0}=2 e_{0}+e_{1}+3 e_{2}+4 e_{3}$ and $P_{1}=e_{0}+3 e_{1}+4 e_{2}+$ 
$7 e_{3}$ in (1.5), then $\left\{P_{n}\right\}$ is the same as the generalized Lucas quaternion sequence considered in [14].

In this paper we introduce the generalized bi-periodic Fibonacci and Lucas quaternion sequences which include $\left\{P_{n}\right\}$ and $\left\{U_{n}\right\}$ as special cases. For those quaternions, we derive the generating functions, Binet's formulas and Catalan's identities.

\section{MAIN RESULTS}

\subsection{Generalized bi-periodic Fibonacci quaternion}

Consider the generalized bi-perioodic Fibonacci sequence $\left\{q_{n}\right\}$ defined by Sahin [16] and Yayenie [21] as

$$
q_{0}=0, q_{1}=1, q_{n}=\left\{\begin{array}{ll}
a q_{n-1}+c q_{n-2}, & \text { if } n \text { is even } \\
b q_{n-1}+d q_{n-2}, & \text { if } n \text { is odd }
\end{array} \quad(n \geq 2) .\right.
$$

The Binet's formula for $\left\{q_{n}\right\}$ is given by [21]

$$
q_{n}=\frac{a^{\zeta(n+1)}}{(a b)^{\left\lfloor\frac{n}{2}\right\rfloor}}\left(\frac{\alpha^{\left\lfloor\frac{n}{2}\right\rfloor}(\alpha+d-c)^{n-\left\lfloor\frac{n}{2}\right\rfloor}-\beta^{\left\lfloor\frac{n}{2}\right\rfloor}(\beta+d-c)^{n-\left\lfloor\frac{n}{2}\right\rfloor}}{\alpha-\beta}\right),
$$

where $\alpha(>0)$ and $\beta(<0)$ are roots of the equation $x^{2}-(a b+c-d) x-a b d=0$.

Definition 1. We define the generalized bi-periodic Fibonacci quaternion sequence $\left\{Q_{n}\right\}$ by

$$
Q_{n}=q_{n} e_{0}+q_{n+1} e_{1}+q_{n+2} e_{2}+q_{n+3} e_{3},
$$

where $q_{n}$ is the $n$th generalized bi-periodic Fibonacci number.

If $c=d=1$, then $\left\{Q_{n}\right\}$ becomes the bi-periodic Fibonacci quaternion sequence given in (1.5).

If $a=b=1$ and $c=d=2$, then $\left\{Q_{n}\right\}$ becomes the Jacobsthal quaternion sequence defined in [17].

In the rest of the paper, we will use the following identities [21] whenever necessary: (i) $\alpha+\beta=a b+c-d$, (ii) $\alpha \beta=-a b d$, (iii) $\alpha(\alpha+d-c)=a b(\alpha+d)$, (iv) $\beta(\beta+d-c)=a b(\beta+d),(\mathrm{v})(\alpha+d)(\beta+d)=c d$.

Theorem 1 (Generating function). The generating function for the generalized bi-periodic Fibonacci quaternion sequence is

$$
G(x)=\frac{\left(1-(a b+d) x^{2}+b c x^{3}\right) Q_{0}+x\left(1+a x-c x^{2}\right) Q_{1}}{1-(a b+c+d) x^{2}+c d x^{4}} .
$$

Proof. We can show that $\left\{Q_{n}\right\}$ satisfies the same recurrence relation as $\left\{q_{n}\right\}$ with the initial condition

$$
\begin{aligned}
Q_{0} & =q_{0} e_{0}+q_{1} e_{1}+q_{2} e_{2}+q_{3} e_{3} \\
& =e_{1}+a e_{2}+(a b+d) e_{3},
\end{aligned}
$$




$$
\begin{aligned}
Q_{1} & =q_{1} e_{0}+q_{2} e_{1}+q_{3} e_{2}+q_{4} e_{3} \\
& =e_{0}+a e_{1}+(a b+d) e_{2}+a(a b+c+d) e_{3},
\end{aligned}
$$

and

$$
\begin{aligned}
Q_{2 n} & =(a b+c+d) Q_{2 n-2}-c d Q_{2 n-4}, \\
Q_{2 n+1} & =(a b+c+d) Q_{2 n-1}-c d Q_{2 n-3} .
\end{aligned}
$$

Then, proceeding as in the proof of [21, Theorem 7], we can obtain (2.4).

If $a=b$ and $c=d$, then

$$
\begin{aligned}
G(x) & =\frac{(1-a x) Q_{0}+x Q_{1}}{1-a x-c x^{2}} \\
& =\frac{x e_{0}+e_{1}+(a+x) e_{2}+\left(a^{2}+c+a c x\right) e_{3}}{1-a x-c x^{2}} .
\end{aligned}
$$

Hence, for $a=b=c=d=1$, we get the generating function for the Fibonacci quaternion

$$
G(x)=\frac{x e_{0}+e_{1}+(1+x) e_{2}+(2+x) e_{3}}{1-x-x^{2}},
$$

as in [10], and, for $a=b=k$ and $c=d=1$, we obtain the generating function for the $k$-Fibonacci quaternion

$$
G(x)=\frac{x e_{0}+e_{1}+(k+x) e_{2}+\left(k^{2}+1+k x\right) e_{3}}{1-k x-x^{2}},
$$

which is given in [15].

Theorem 2 (Binet's formula). The Binet's formula for the generalized bi-periodic Fibonacci quaternion sequence is

$$
Q_{n}= \begin{cases}\frac{1}{(a b)^{\left\lfloor\frac{n}{2}\right\rfloor}}\left(\frac{\alpha_{e} \alpha^{\left\lfloor\frac{n}{2}\right\rfloor}(\alpha+d-c)^{n-\left\lfloor\frac{n}{2}\right\rfloor}-\beta_{e} \beta^{\left\lfloor\frac{n}{2}\right\rfloor}(\beta+d-c)^{n-\left\lfloor\frac{n}{2}\right\rfloor}}{\alpha-\beta}\right), & \text { if } n \text { is even } \\ \frac{1}{(a b)^{\left\lfloor\frac{n}{2}\right\rfloor}}\left(\frac{\alpha_{o} \alpha^{\left\lfloor\frac{n}{2}\right\rfloor}(\alpha+d-c)^{n-\left\lfloor\frac{n}{2}\right\rfloor}-\beta_{o} \beta^{\left\lfloor\frac{n}{2}\right\rfloor}(\beta+d-c)^{n-\left\lfloor\frac{n}{2}\right\rfloor}}{\alpha-\beta}\right), & \text { if } n \text { is odd }\end{cases}
$$

where

$$
\begin{aligned}
& \alpha_{e}=\sum_{l=0}^{3} \frac{a^{\zeta(l+1)}}{(a b)^{\left\lfloor\frac{l}{2}\right\rfloor}} \alpha^{\left\lfloor\frac{l}{2}\right\rfloor}(\alpha+d-c)^{\left\lfloor\frac{l+1}{2}\right\rfloor} e_{l}, \\
& \beta_{e}=\sum_{l=0}^{3} \frac{a^{\zeta(l+1)}}{(a b)^{\left\lfloor\frac{l}{2}\right\rfloor}} \beta^{\left\lfloor\frac{l}{2}\right\rfloor}(\beta+d-c)^{\left\lfloor\frac{l+1}{2}\right\rfloor} e_{l}, \\
& \alpha_{o}=\sum_{l=0}^{3} \frac{a^{\zeta(l)}}{(a b)^{\left\lfloor\frac{l+1}{2}\right\rfloor}} \alpha^{\left\lfloor\frac{l+1}{2}\right\rfloor}(\alpha+d-c)^{\left\lfloor\frac{l}{2}\right\rfloor} e_{l},
\end{aligned}
$$




$$
\beta_{o}=\sum_{l=0}^{3} \frac{a^{\zeta(l)}}{(a b)^{\left\lfloor\frac{l+1}{2}\right\rfloor}} \beta^{\left\lfloor\frac{l+1}{2}\right\rfloor}(\beta+d-c)^{\left\lfloor\frac{l}{2}\right\rfloor} e_{l} .
$$

Proof. Firstly we note that $\left\lfloor\frac{n}{2}\right\rfloor=\frac{n-\zeta(n)}{2}$.

¿From (2.2) and (2.3), we have

$$
\begin{aligned}
& Q_{n}=\frac{a^{\zeta(n+1)}}{(a b)^{\left\lfloor\frac{n}{2}\right\rfloor}}\left(\frac{\alpha^{\left\lfloor\frac{n}{2}\right\rfloor}(\alpha+d-c)^{n-\left\lfloor\frac{n}{2}\right\rfloor}-\beta^{\left\lfloor\frac{n}{2}\right\rfloor}(\beta+d-c)^{n-\left\lfloor\frac{n}{2}\right\rfloor}}{\alpha-\beta}\right) e_{0} \\
& +\frac{a^{\zeta(n)}}{(a b)^{\zeta(n)}(a b)^{\left\lfloor\frac{n}{2}\right\rfloor}}\left(\frac{\alpha^{\zeta(n)}(\alpha+d-c)^{\zeta(n+1)} \alpha^{\left\lfloor\frac{n}{2}\right\rfloor}(\alpha+d-c)^{n-\left\lfloor\frac{n}{2}\right\rfloor}}{\alpha-\beta}\right. \\
& \left.-\frac{\beta^{\zeta(n)}(\beta+d-c)^{\zeta(n+1)} \beta^{\left\lfloor\frac{n}{2}\right\rfloor}(\beta+d-c)^{n-\left\lfloor\frac{n}{2}\right\rfloor}}{\alpha-\beta}\right) e_{1} \\
& +\frac{a^{\zeta(n+1)}}{a b(a b)^{\left\lfloor\frac{n}{2}\right\rfloor}}\left(\frac{\alpha(\alpha+d-c) \alpha^{\left\lfloor\frac{n}{2}\right\rfloor}(\alpha+d-c)^{n-\left\lfloor\frac{n}{2}\right\rfloor}}{\alpha-\beta}\right. \\
& \left.-\frac{\beta(\beta+d-c) \beta^{\left\lfloor\frac{n}{2}\right\rfloor}(\beta+d-c)^{n-\left\lfloor\frac{n}{2}\right\rfloor}}{\alpha-\beta}\right) e_{2} \\
& +\frac{a^{\zeta(n)}}{(a b)^{1+\zeta(n)}(a b)^{\left\lfloor\frac{n}{2}\right\rfloor}}\left(\frac{\alpha^{1+\zeta(n)}(\alpha+d-c)^{1+\zeta(n+1)} \alpha^{\left\lfloor\frac{n}{2}\right\rfloor}(\alpha+d-c)^{n-\left\lfloor\frac{n}{2}\right\rfloor}}{\alpha-\beta}\right. \\
& \left.-\frac{\beta^{1+\zeta(n)}(\beta+d-c)^{1+\zeta(n+1)} \beta^{\left\lfloor\frac{n}{2}\right\rfloor}(\beta+d-c)^{n-\left\lfloor\frac{n}{2}\right\rfloor}}{\alpha-\beta}\right) e_{3},
\end{aligned}
$$

or

$$
Q_{n}=\frac{1}{(a b)^{\left\lfloor\frac{n}{2}\right\rfloor}}\left(\frac{\alpha_{n} \alpha^{\left\lfloor\frac{n}{2}\right\rfloor}(\alpha+d-c)^{n-\left\lfloor\frac{n}{2}\right\rfloor}-\beta_{n} \beta^{\left\lfloor\frac{n}{2}\right\rfloor}(\beta+d-c)^{n-\left\lfloor\frac{n}{2}\right\rfloor}}{\alpha-\beta}\right),
$$

where

$$
\begin{aligned}
\alpha_{n} & =a^{\zeta(n+1)} e_{0}+\frac{a^{\zeta(n)} \alpha^{\zeta(n)}(\alpha+d-c)^{\zeta(n+1)}}{(a b)^{\zeta(n)}} e_{1} \\
& +\frac{a^{\zeta(n+1)} \alpha(\alpha+d-c)}{a b} e_{2}+\frac{a^{\zeta(n)} \alpha^{1+\zeta(n)}(\alpha+d-c)^{1+\zeta(n+1)}}{(a b)^{1+\zeta(n)}} e_{3}, \\
\beta_{n} & =a^{\zeta(n+1)} e_{0}+\frac{a^{\zeta(n)} \beta^{\zeta(n)}(\beta+d-c)^{\zeta(n+1)}}{(a b)^{\zeta(n)}} e_{1} \\
& +\frac{a^{\zeta(n+1)} \beta(\beta+d-c)}{a b} e_{2}+\frac{a^{\zeta(n)} \beta^{1+\zeta(n)}(\beta+d-c)^{1+\zeta(n+1)}}{(a b)^{1+\zeta(n)}} e_{3} .
\end{aligned}
$$


If $n$ is even, then

$$
\begin{aligned}
\alpha_{n} & =a e_{0}+(\alpha+d-c) e_{1}+\frac{a \alpha(\alpha+d-c)}{a b} e_{2}+\frac{\alpha(\alpha+d-c)^{2}}{a b} e_{3} \\
& =\sum_{l=0}^{3} \frac{a^{\zeta(l+1)}}{(a b)^{\left\lfloor\frac{l}{2}\right\rfloor}} \alpha^{\left\lfloor\frac{l}{2}\right\rfloor}(\alpha+d-c)^{\left\lfloor\frac{l+1}{2}\right\rfloor} e_{l}, \\
\beta_{n} & =a e_{0}+(\beta+d-c) e_{1}+\frac{a \beta(\beta+d-c)}{a b} e_{2}+\frac{\beta(\beta+d-c)^{2}}{a b} e_{3} \\
& =\sum_{l=0}^{3} \frac{a^{\zeta(l+1)}}{(a b)^{\left\lfloor\frac{l}{2}\right\rfloor}} \beta^{\left\lfloor\frac{l}{2}\right\rfloor}(\beta+d-c)^{\left\lfloor\frac{l+1}{2}\right\rfloor} e_{l} .
\end{aligned}
$$

Similarly, if $n$ is odd, then

$$
\begin{aligned}
& \alpha_{n}=\sum_{l=0}^{3} \frac{a^{\zeta(l)}}{(a b)^{\left\lfloor\frac{l+1}{2}\right\rfloor}} \alpha^{\left\lfloor\frac{l+1}{2}\right\rfloor}(\alpha+d-c)^{\left\lfloor\frac{l}{2}\right\rfloor} e_{l}, \\
& \beta_{n}=\sum_{l=0}^{3} \frac{a^{\zeta(l)}}{(a b)^{\left\lfloor\frac{l+1}{2}\right\rfloor}} \beta^{\left\lfloor\frac{l+1}{2}\right\rfloor}(\beta+d-c)^{\left\lfloor\frac{l}{2}\right\rfloor} e_{l},
\end{aligned}
$$

and the proof is completed.

If $c=d=1$, then (2.5) becomes the Binet's formula for the bi-periodic Fibonacci quaternion given in (1.6).

Theorem 3 (Catalan's identity). The Catalan's identity for the generalized biperiodic Fibonacci quaternion sequence is

$$
\begin{aligned}
& Q_{n}^{2}-Q_{n+2 r} Q_{n-2 r}
\end{aligned}
$$

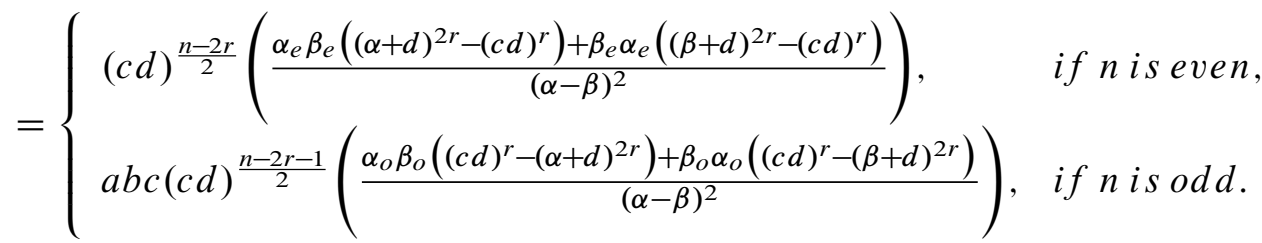

Proof. Firstly, assume that $n$ is even, and let

$$
\begin{aligned}
& X_{1}=(\alpha-\beta)^{2}(a b)^{n} Q_{n}^{2}, \\
& X_{2}=(\alpha-\beta)^{2}(a b)^{n} Q_{n+2 r} Q_{n-2 r} .
\end{aligned}
$$

Then

$$
\begin{aligned}
X_{1} & =\left(\alpha_{e} \alpha^{\frac{n}{2}}(\alpha+d-c)^{\frac{n}{2}}-\beta_{e} \beta^{\frac{n}{2}}(\beta+d-c)^{\frac{n}{2}}\right)^{2} \\
& =\alpha_{e}^{2} \alpha^{n}(\alpha+d-c)^{n}+\beta_{e}^{2} \beta^{n}(\beta+d-c)^{n}
\end{aligned}
$$




$$
\begin{aligned}
& -\left(\alpha_{e} \beta_{e}+\beta_{e} \alpha_{e}\right) \alpha^{\frac{n}{2}}(\alpha+d-c)^{\frac{n}{2}} \beta^{\frac{n}{2}}(\beta+d-c)^{\frac{n}{2}}, \\
& =\alpha_{e}^{2} \alpha^{n}(\alpha+d-c)^{n}+\beta_{e}^{2} \beta^{n}(\beta+d-c)^{n} \\
& -\left(\alpha_{e} \beta_{e}+\beta_{e} \alpha_{e}\right)(a b)^{n}(\alpha+d)^{\frac{n}{2}}(\beta+d)^{\frac{n}{2}}, \\
& =\alpha_{e}^{2} \alpha^{n}(\alpha+d-c)^{n}+\beta_{e}^{2} \beta^{n}(\beta+d-c)^{n} \\
& -\left(\alpha_{e} \beta_{e}+\beta_{e} \alpha_{e}\right)(a b)^{n}(c d)^{\frac{n}{2}},
\end{aligned}
$$

and

$$
\begin{aligned}
X_{2} & =\left(\alpha_{e} \alpha^{\frac{n+2 r}{2}}(\alpha+d-c)^{\frac{n+2 r}{2}}-\beta_{e} \beta^{\frac{n+2 r}{2}}(\beta+d-c)^{\frac{n+2 r}{2}}\right) \\
& \times\left(\alpha_{e} \alpha^{\frac{n-2 r}{2}}(\alpha+d-c)^{\frac{n-2 r}{2}}-\beta_{e} \beta^{\frac{n-2 r}{2}}(\beta+d-c)^{\frac{n-2 r}{2}}\right) \\
& =\alpha_{e}^{2} \alpha^{n}(\alpha+d-c)^{n}+\beta_{e}^{2} \beta^{n}(\beta+d-c)^{n} \\
& -\alpha_{e} \beta_{e} \alpha^{\frac{n+2 r}{2}}(\alpha+d-c)^{\frac{n+2 r}{2}} \beta^{\frac{n-2 r}{2}}(\beta+d-c)^{\frac{n-2 r}{2}} \\
& -\beta_{e} \alpha_{e} \alpha^{\frac{n-2 r}{2}}(\alpha+d-c)^{\frac{n-2 r}{2}} \beta^{\frac{n+2 r}{2}}(\beta+d-c)^{\frac{n+2 r}{2}}, \\
& =\alpha_{e}^{2} \alpha^{n}(\alpha+d-c)^{n}+\beta_{e}^{2} \beta^{n}(\beta+d-c)^{n} \\
& -\alpha_{e} \beta_{e}(a b)^{n}(\alpha+d)^{\frac{n+2 r}{2}}(\beta+d)^{\frac{n-2 r}{2}} \\
& -\beta_{e} \alpha_{e}(a b)^{n}(\alpha+d)^{\frac{n-2 r}{2}}(\beta+d)^{\frac{n+2 r}{2}}, \\
& =\alpha_{e}^{2} \alpha^{n}(\alpha+d-c)^{n}+\beta_{e}^{2} \beta^{n}(\beta+d-c)^{n} \\
& -\alpha_{e} \beta_{e}(a b)^{n}(c d)^{\frac{n-2 r}{2}}(\alpha+d)^{2 r} \\
& -\beta_{e} \alpha_{e}(a b)^{n}(c d)^{\frac{n-2 r}{2}}(\beta+d)^{2 r} .
\end{aligned}
$$

Hence

$$
\begin{aligned}
X_{1}-X_{2} & =\alpha_{e} \beta_{e}(a b)^{n}(c d)^{\frac{n-2 r}{2}}\left((\alpha+d)^{2 r}-(c d)^{r}\right) \\
& +\beta_{e} \alpha_{e}(a b)^{n}(c d)^{\frac{n-2 r}{2}}\left((\beta+d)^{2 r}-(c d)^{r}\right),
\end{aligned}
$$

and the proof is completed for the case where $n$ is even.

When $n$ is odd, we can proceed similarly, and details are omitted.

If $c=d=1$, then $\alpha^{2}=a b(\alpha+1)$ and

$$
\begin{aligned}
(\alpha+1)^{2 r}-1 & =\frac{(a b)^{2 r}(\alpha+1)^{2 r}-(a b)^{2 r}}{(a b)^{2 r}} \\
& =\frac{\alpha^{4 r}-(a b)^{2 r}}{(a b)^{2 r}} .
\end{aligned}
$$


Similarly

$$
(\beta+1)^{2 r}-1=\frac{\beta^{4 r}-(a b)^{2 r}}{(a b)^{2 r}},
$$

and Theorem 3 reduces to [19, Theorem 5].

\subsection{Generalized bi-periodic Lucas quaternion}

Consider the generalized bi-periodic Lucas sequence $\left\{v_{n}\right\}$ defined by Bilgici [2] as

$$
v_{0}=\frac{d+1}{d}, v_{1}=a, \quad v_{n}=\left\{\begin{array}{ll}
b v_{n-1}+d v_{n-2}, & \text { if } n \text { is even } \\
a v_{n-1}+c v_{n-2}, & \text { if } n \text { is odd }
\end{array}(n \geq 2) .\right.
$$

The Binet's formula for $\left\{v_{n}\right\}$ is given by [2]

$$
v_{n}=\frac{a^{\zeta(n)}}{(a b)^{\left\lfloor\frac{n-1}{2}\right\rfloor}}\left(\frac{(\alpha+d+1) \alpha^{\left\lfloor\frac{n-1}{2}\right\rfloor}(\alpha+d-c)^{\left\lfloor\frac{n}{2}\right\rfloor}-(\beta+d+1) \beta^{\left\lfloor\frac{n-1}{2}\right\rfloor}(\beta+d-c)^{\left\lfloor\frac{n}{2}\right\rfloor}}{\alpha-\beta}\right),
$$

where $\alpha$ and $\beta$ are as defined in (2.2).

Definition 2. The generalized bi-periodic Lucas quaternion sequence $\left\{V_{n}\right\}$ is defined by

$$
V_{n}=v_{n} e_{0}+v_{n+1} e_{1}+v_{n+2} e_{2}+v_{n+3} e_{3},
$$

where $v_{n}$ is the $n$th generalized bi-periodic Lucas number.

If $c=d=1,\left\{V_{n}\right\}$ becomes the bi-periodic Lucas quaternion sequence given in (1.7).

Theorem 4 (Generating function). The generating function for the generalized bi-periodic Lucas quaternion sequence is

$$
H(x)=\frac{\left(1-(a b+c) x^{2}+a d x^{3}\right) V_{0}+x\left(1+b x-d x^{2}\right) V_{1}}{1-(a b+c+d) x^{2}+c d x^{4}} .
$$

Proof. Replacing $Q_{0}, Q_{1}, a, b, c$ and $d$ by $V_{0}, V_{1}, b, a, d$ and $c$ in (2.4), we obtain (2.10).

$$
\text { If } a=b \text { and } c=d \text {, then }
$$

$$
H(x)=\begin{array}{r}
\frac{(1-a x) V_{0}+x V_{1}}{1-a x-c x^{2}} \\
=\frac{\frac{c+1-a x}{c} e_{0}+(a+(c+1) x) e_{1}+\left(a^{2}+c+1+a c x\right) e_{2}}{1-a x-c x^{2}} \\
+\frac{\left(a^{3}+2 a c+a+\left(a^{2}+c^{2}+1\right) x\right) e_{3}}{1-a x-c x^{2}} .
\end{array}
$$


Hence, for $a=b=k$ and $c=d=1$, we obtain the generating function for the $k$-Lucas quaternion

$$
H(x)=\frac{(2-k x) e_{0}+(k+2 x) e_{1}+\left(k^{2}+2+k x\right) e_{2}+\left(k^{3}+3 k+\left(k^{2}+2\right) x\right) e_{3}}{1-k x-x^{2}},
$$

as in [15].

Theorem 5 (Binet's formula). The Binet's formula for the generalized bi-periodic Lucas quaternion sequence is

$$
V_{n}= \begin{cases}\frac{1}{\left.(a b)^{\left\lfloor^{\lfloor-1}\right.}\right\rfloor} V_{n e}, & \text { if } n \text { is even } \\ \frac{1}{(a b)^{\left\lfloor^{\frac{n-1}{2}}\right\rfloor}} V_{n o}, & \text { if } n \text { is odd }\end{cases}
$$

where

$$
\begin{aligned}
& V_{n e}=\frac{\alpha_{o}(\alpha+d+1) \alpha^{\left\lfloor\frac{n-1}{2}\right\rfloor}(\alpha+d-c)^{\left\lfloor\frac{n}{2}\right\rfloor}-\beta_{o}(\beta+d+1) \beta^{\left\lfloor\frac{n-1}{2}\right\rfloor}(\beta+d-c)^{\left\lfloor\frac{n}{2}\right\rfloor}}{\alpha-\beta}, \\
& V_{n o}=\frac{\alpha_{e}(\alpha+d+1) \alpha^{\left\lfloor\frac{n-1}{2}\right\rfloor}(\alpha+d-c)^{\left\lfloor\frac{n}{2}\right\rfloor}-\beta_{e}(\beta+d+1) \beta^{\left\lfloor\frac{n-1}{2}\right\rfloor}(\beta+d-c)^{\left\lfloor\frac{n}{2}\right\rfloor}}{\alpha-\beta},
\end{aligned}
$$

with $\alpha_{e}, \beta_{e}, \alpha_{o}$ and $\beta_{o}$ as defined in (2.5).

Proof. Using the Binet's formula for $\left\{v_{n}\right\}$ and proceeding as in the proof of Theorem 2, we can easily obtain (2.11).

If $c=d=1$, then

$$
\begin{aligned}
(\alpha+2) \alpha^{\left\lfloor\frac{n-1}{2}\right\rfloor+\left\lfloor\frac{n}{2}\right\rfloor} & =(\alpha+2) \alpha^{n-1} \\
& =\left(1+\frac{2}{\alpha}\right) \alpha^{n} \\
& =\left(1-\frac{2 \beta}{a b}\right) \alpha^{n} \\
& =\frac{(\alpha-\beta) \alpha^{n}}{a b},
\end{aligned}
$$

and

$$
\begin{aligned}
(\beta+2) \beta^{\left\lfloor\frac{n-1}{2}\right\rfloor+\left\lfloor\frac{n}{2}\right\rfloor} & =(\beta+2) \beta^{n-1} \\
& =\left(1+\frac{2}{\beta}\right) \beta^{n} \\
& =\left(1-\frac{2 \alpha}{a b}\right) \beta^{n} \\
& =\frac{(\beta-\alpha) \beta^{n}}{a b} .
\end{aligned}
$$


Hence (2.11) reduces to the Binet's formula for the bi-periodic Lucas quaternion given in (1.8).

We verify (2.11) for $n=1$. From (2.7) and the definition of $\left\{V_{n}\right\}$, we have

$$
\begin{aligned}
V_{1} & =v_{1} e_{0}+v_{2} e_{1}+v_{3} e_{2}+v_{4} e_{3} \\
& =a e_{0}+(a b+d+1) e_{1}+a(a b+c+d+1) e_{2} \\
& +\left(a^{2} b^{2}+a b c+2 a b d+a b+d^{2}+d\right) e_{3} .
\end{aligned}
$$

On the other hand, if $n=1$, then (2.11) becomes

$$
V_{1}=\frac{\alpha_{e}(\alpha+d+1)-\beta_{e}(\beta+d+1)}{\alpha-\beta} .
$$

In this case, $\alpha_{e}$ and $\beta_{e}$ respectively can be written as

$$
\begin{aligned}
& \alpha_{e}=a e_{o}+(\alpha+d-c) e_{1}+a(\alpha+d) e_{2}+(\alpha+d)(\alpha+d-c) e_{3}, \\
& \beta_{e}=a e_{o}+(\beta+d-c) e_{1}+a(\beta+d) e_{2}+(\beta+d)(\beta+d-c) e_{3} .
\end{aligned}
$$

Let

$$
\alpha_{e}(\alpha+d+1)-\beta_{e}(\beta+d+1)=E_{0} e_{0}+E_{1} e_{1}+E_{2} e_{2}+E_{3} e_{3} .
$$

Then

$$
\begin{aligned}
E_{0} & =a(\alpha-\beta), \\
E_{1} & =(\alpha+d+1)(\alpha+d-c)-(\beta+d+1)(\beta+d-c) \\
& =(\alpha+d)^{2}-(\beta+d)^{2}-(c-1)(\alpha-\beta) \\
& =(\alpha+\beta+2 d)(\alpha-\beta)-(c-1)(\alpha-\beta) \\
& =(a b+d+1)(\alpha-\beta), \\
E_{2} & =a((\alpha+d)(\alpha+d-c)-(\beta+d)(\beta+d-c)) \\
& =a\left((\alpha+d)^{2}-(\beta+d)^{2}+(\alpha-\beta)\right) \\
& =a((\alpha+\beta+2 d)(\alpha-\beta)+(\alpha-\beta)) \\
& =a(a b+c+d+1)(\alpha-\beta), \\
E_{3} & =(\alpha+d)(\alpha+d+1)(\alpha+d-c)-(\beta+d)(\beta+d+1)(\beta+d-c) \\
& =(\alpha+d)^{3}-(\beta+d)^{3}-(c-1)\left((\alpha+d)^{2}-(\beta+d)^{2}\right)-c(\alpha-\beta) \\
& =\left((\alpha+\beta+2 d)^{2}-(\alpha+d)(\beta+d)\right)(\alpha-\beta) \\
& -(c-1)(\alpha+\beta+2 d)(\alpha-\beta)-c(\alpha-\beta) \\
& =\left((a b+c+d)^{2}-c d\right)(\alpha-\beta)-(c-1)(a b+c+d)(\alpha-\beta)-c(\alpha-\beta) \\
& =\left(a^{2} b^{2}+a b c+2 a b d+a b+d^{2}+d\right)(\alpha-\beta) .
\end{aligned}
$$

Hence (2.11) is true for $n=1$. 
Theorem 6 (Catalan's identity). The Catalan's identity for the generalized biperiodic Lucas quaternion sequence is

$$
\begin{aligned}
& V_{n}^{2}-V_{n+2 r} V_{n-2 r} \\
& =\left\{\begin{array}{l}
\frac{(c d)^{\frac{n-2 r}{2}}\left(d \alpha-\beta-c d+d^{2}\right)\left(d \beta-\alpha-c d+d^{2}\right)}{d^{2}} \\
\times\left(\frac{\alpha_{o} \beta_{o}\left((\alpha+d)^{2 r}-(c d)^{r}\right)+\beta_{o} \alpha_{o}\left((\beta+d)^{2 r}-(c d)^{r}\right)}{(\alpha-\beta)^{2}}\right), \\
\frac{(c d)^{\frac{n-2 r-1}{2}}\left(d \alpha-\beta-c d+d^{2}\right)\left(d \beta-\alpha-c d+d^{2}\right)}{a b d} \\
\times\left(\frac{\alpha_{e} \beta_{e}\left((c d)^{r}-(\alpha+d)^{2 r}\right)+\beta_{e} \alpha_{e}\left((c d)^{r}-(\beta+d)^{2 r}\right)}{(\alpha-\beta)^{2}}\right),
\end{array}\right.
\end{aligned}
$$

Proof. Assume that $n$ is even, and let

$$
\begin{aligned}
& Y_{1}=(\alpha-\beta)^{2}(a b)^{n-2} V_{n}^{2}, \\
& Y_{2}=(\alpha-\beta)^{2}(a b)^{n-2} V_{n+2 r} V_{n-2 r} .
\end{aligned}
$$

Then

$$
\begin{aligned}
Y_{1} & =\left(\alpha_{o}(\alpha+d+1) \alpha^{\frac{n-2}{2}}(\alpha+d-c)^{\frac{n}{2}}-\beta_{o}(\beta+d+1) \beta^{\frac{n-2}{2}}(\beta+d-c)^{\frac{n}{2}}\right)^{2} \\
& =\alpha_{o}^{2}(\alpha+d+1)^{2} \alpha^{n-2}(\alpha+d-c)^{n}+\beta_{o}^{2}(\beta+d+1)^{2} \beta^{n-2}(\beta+d-c)^{n} \\
& -\left(\alpha_{o} \beta_{o}+\beta_{o} \alpha_{o}\right)(\alpha+d+1)(\beta+d+1) \alpha^{\frac{n-2}{2}}(\alpha+d-c)^{\frac{n}{2}} \beta^{\frac{n-2}{2}}(\beta+d-c)^{\frac{n}{2}},
\end{aligned}
$$

and

$$
\begin{aligned}
Y_{2} & =\left(\alpha_{o}(\alpha+d+1) \alpha^{\frac{n+2 r-2}{2}}(\alpha+d-c)^{\frac{n+2 r}{2}}-\beta_{o}(\beta+d+1) \beta^{\frac{n+2 r-2}{2}}(\beta+d-c)^{\frac{n+2 r}{2}}\right) \\
& \times\left(\alpha_{o}(\alpha+d+1) \alpha^{\frac{n-2 r-2}{2}}(\alpha+d-c)^{\frac{n-2 r}{2}}-\beta_{o}(\beta+d+1) \beta^{\frac{n-2 r-2}{2}}(\beta+d-c)^{\frac{n-2 r}{2}}\right), \\
& =\alpha_{o}^{2}(\alpha+d+1)^{2} \alpha^{n-2}(\alpha+d)^{n}+\beta_{o}^{2}(\beta+d+1)^{2} \beta^{n-2}(\beta+d-c)^{n} \\
& -\alpha_{o} \beta_{o}(\alpha+d+1)(\beta+d+1) \alpha^{\frac{n+2 r-2}{2}}(\alpha+d-c)^{\frac{n+2 r}{2}} \beta^{\frac{n-2 r-2}{2}}(\beta+d-c)^{\frac{n-2 r}{2}}, \\
& -\beta_{o} \alpha_{o}(\alpha+d+1)(\beta+d+1) \alpha^{\frac{n-2 r-2}{2}}(\alpha+d-c)^{\frac{n-2 r}{2}} \beta^{\frac{n+2 r-2}{2}}(\beta+d-c)^{\frac{n+2 r}{2}} .
\end{aligned}
$$

Hence

$$
Y_{1}-Y_{2}=\alpha_{o} \beta_{o} A_{1}+\beta_{o} \alpha_{o} A_{2}
$$

where

$$
\begin{aligned}
A_{1} & =(\alpha+d+1)(\beta+d+1)\left(\alpha^{\frac{n+2 r-2}{2}}(\alpha+d-c)^{\frac{n+2 r}{2}} \beta^{\frac{n-2 r-2}{2}}(\beta+d-c)^{\frac{n-2 r}{2}}\right. \\
& \left.-\alpha^{\frac{n-2}{2}}(\alpha+d-c)^{\frac{n}{2}} \beta^{\frac{n-2}{2}}(\beta+d-c)^{\frac{n}{2}}\right) \\
A_{2} & =(\alpha+d+1)(\beta+d+1)\left(\alpha^{\frac{n-2 r-2}{2}}(\alpha+d-c)^{\frac{n-2 r}{2}} \beta^{\frac{n+2 r-2}{2}}(\beta+d-c)^{\frac{n+2 r}{2}}\right. \\
& \left.-\alpha^{\frac{n-2}{2}}(\alpha+d-c)^{\frac{n}{2}} \beta^{\frac{n-2}{2}}(\beta+d-c)^{\frac{n}{2}}\right) .
\end{aligned}
$$


Since $\alpha \beta=-a b d$ and $\alpha+\beta=a b+c-d$, we have

Similarly

$$
\begin{aligned}
(\alpha+d+1) & =\left(1+\frac{d+1}{\alpha}\right) \alpha \\
& =\left(1-\frac{(d+1) \beta}{a b d}\right) \alpha \\
& =\left(\frac{(\alpha+\beta) d-d(c-d)-(d+1) \beta}{a b d}\right) \alpha \\
& =\frac{\left(d \alpha-\beta-c d+d^{2}\right) \alpha}{a b d} .
\end{aligned}
$$

$$
(\beta+d+1)=\frac{\left(d \beta-\alpha-c d+d^{2}\right) \beta}{a b d} .
$$

Then

$$
\begin{aligned}
A_{1} & =\frac{\left(d \alpha-\beta-c d+d^{2}\right)\left(d \beta-\alpha-c d+d^{2}\right) \alpha^{\frac{n}{2}}(\alpha+d-c)^{\frac{n}{2}} \beta^{\frac{n}{2}}(\beta+d-c)^{\frac{n}{2}}}{(a b d)^{2}} \\
& \times\left(\frac{\alpha^{r}(\alpha+d-c)^{r}}{\beta^{r}(\beta+d-c)^{r}}-1\right) \\
& =\frac{(a b)^{n-2}(c d)^{\frac{n}{2}}\left(d \alpha-\beta-c d+d^{2}\right)\left(d \beta-\alpha-c d+d^{2}\right)}{d^{2}} \\
& \times \frac{\alpha^{2 r}(\alpha+d-c)^{2 r}-\alpha^{r}(\alpha+d-c)^{r} \beta^{r}(\beta+d-c)^{r}}{\alpha^{r}(\alpha+d-c)^{r} \beta^{r}(\beta+d-c)^{r}} \\
& =\frac{(a b)^{n-2}(c d)^{\frac{n-2 r}{2}}\left(d \alpha-\beta-c d+d^{2}\right)\left(d \beta-\alpha-c d+d^{2}\right)\left((\alpha+d)^{2 r}-(c d)^{r}\right)}{d^{2}} .
\end{aligned}
$$

Similarly we have

$$
A_{2}=\frac{(a b)^{n-2}(c d)^{\frac{n-2 r}{2}}\left(d \alpha-\beta-c d+d^{2}\right)\left(d \beta-\alpha-c d+d^{2}\right)\left((\beta+d)^{2 r}-(c d)^{r}\right)}{d^{2}},
$$

and the proof is completed for the case where $n$ is even.

Using the same procedure, we can also prove (2.12) for the case where $n$ is odd.

$$
\text { If } c=d=1 \text {, then Theorem } 6 \text { reduces to [18, Theorem 5]. }
$$

\section{CONCLUSIONS}

In this paper we introduced the generalized bi-periodic Fibonacci and Lucas quaternions which are the further generalizations of the bi-periodic Fibonacci and Lucas quaternions considered in the literature. For those quaternions, we obtained the generating functions, Binet's formulas and Catalan's identities. 


\section{ACKNOWLEDGEMENT}

The author thanks to the anonymous reviewer for helpful comments which led to improved presentation of the paper.

\section{REFERENCES}

[1] S. L. Adler, Quaternionic quantum mechanics and quantum fields. New York: Oxford University Press, 1994.

[2] G. Bilgici, "Two generalizations of Lucas sequence.” Appl. Math. Comp., vol. 245, pp. 526-538, 2014, doi: 10.1016/j.amc.2014.07.111.

[3] P. Catarino, "The modified Pell and the modified $k$-Pell quaternions and octonions." Adv. Appl. Clifford Algebras, vol. 26, no. 2, pp. 577-590, 2016, doi: 10.1007/s00006-015-0611-4.

[4] C. B. Çimen and A. İpek, "On Pell quaternions and Pell-Lucas quaternions." Adv. Appl. Clifford Algebras, vol. 26, pp. 39-51, 2016, doi: 10.1007/s00006-015-0571-8.

[5] E. Edson and O. Yayenie, "A new generalization of Fibonacci sequences and extended Binet's formula." Integers, vol. 9, pp. 639-654, 2009, doi: 10.1515/INTEG.2009.051.

[6] T. A. Ell, N. L. Bihan, and S. J. Sangwine, Quaternion Fourier transforms for signal processing and image processing. John Wiley \& Sons, 2014.

[7] S. Falcón, "On the $k$-Lucas numbers." Int. J. Contempt. Math. Sci., vol. 6, no. 21-24, pp. 10391050, 2011, doi: 10.12988//ijcms.

[8] S. Falcón and A. Plaza, "On the Fibonacci $k$-numbers." Chaos, Solitons and Fractals, vol. 32, no. 5, pp. 1615-1624, 2007, doi: 10.1016//j.chaos.2006.09.022.

[9] K. Gürlebeck and W. Sprössig, Quaternionic and Clifford calculus for physicists and engineers. New York: Wiley, 1997.

[10] S. Halici, "On Fibonacci quaternions." Adv. Appl. Clifford Algebras, vol. 22, no. 2, pp. 321-327, 2012, doi: 10.1007/s00006-011-0317-1.

[11] S. Halici, "On complex Fibonacci quaternions." Adv. Appl. Clifford Algebras, vol. 23, no. 1, pp. 105-112, 2013, doi: 10.1007/s00006-012-0337-5.

[12] S. Halici and A. Karataş, "On a generalization for Fibonacci quaternions." Chaos, Solitons and Fractals, vol. 98, pp. 178-182, 2017, doi: http.//dx.doi.org/10.1016/j.chaos.2017.03.037.

[13] A. F. Horadam, "Complex Fibonacci numbers and Fibonacci quaternions.” Amer. Math. Monthly, vol. 70, no. 3, pp. 289-291, 1963, doi: https://about.jstor.org/terms.

[14] E. Polatli, "A generalization of Fibonacci and Lucas quaternions." Adv. Appl. Clifford Algebras, vol. 26, no. 2, pp. 719-730, 2016, doi: 10.1007/s00006-015-0626-x.

[15] J. L. Ram irez, "Some combinatorial properties of the $k$-Fibonacci and the $k$-Lucas quaternions." An Şt. Univ. Ovidius Constanta, vol. 23, no. 2, pp. 201-212, 2015, doi: 10.1515/auom-2015-0037.

[16] M. Sahin, "The Gelin-Cesàro identity in some conditional sequences." Hacet. J. Math. Stat., vol. 40, no. 6, pp. 855-861, 2011.

[17] A. Szynal-Liana and I. Włoch, "A note on Jacobsthal quaternions." Adv. Appl. Clifford Algebras, vol. 26, no. 1, pp. 441-447, 2016, doi: 10.1007/s00006-015-0622-1.

[18] E. Tan, S. Yilmiz, and M. Sahin, "A note on bi-periodic Fibonacci and Lucas quaternions." Chaos, Solitons and Fractals, vol. 85, pp. 138-142, 2016, doi: http.//dx.doi.org/10.1016/j.chaos.2016.01.025.

[19] E. Tan, S. Yilmiz, and M. Sahin, "On a new generalization of Fibonacci quaternions." Chaos, Solitons and Fractals, vol. 82, pp. 1-4, 2016, doi: http.//dx.doi.org/10.1016/j.chaos.2015.10.021.

[20] J. P. Ward, Quaternions and Cayley numbers: algebra and applications. London: Kluwer Academic Publisher, 1997. 
[21] O. Yayenie, "A note on generalized Fibonacci sequences." Appl. Math. Comp., vol. 217, no. 12, pp. 5603-5611, 2011, doi: 10.1016/j.amc.2010.12.038.

Author's address

Younseok Choo

Hongik University, Department of Electronic and Electrical Engineering, 2639 Sejong-Ro, 30016 Sejong, Republic of Korea

E-mail address: yschoo@hongik.ac.kr 